\title{
Stress field and fracture propagation due to the change of hydraulic pressure
}

\author{
Kurama OKUBO $^{1}$, Hitoshi MIKADA ${ }^{1}$, Tada-noi GOTO ${ }^{1}$ and Junichi TAKEKAWA ${ }^{1}$ \\ ${ }^{1}$ Dept. of Civil and Earth Res. Eng., Kyoto University
}

\begin{abstract}
In the developing of the hydrothermal or shale oil and gas reservoir, hydraulic fracturing method is widely used today. It creates fracture nets around the well and improves the permeability, which enhances the production of those resources. However, it is challenging task to predict the direction and area of the hydraulic fracturing due to the complicated in-situ stress field. Fracturing to the unexpected area might cause induced seismicity or environmental pollution. It is, therefore, quite important to estimate how fractures are creates in the reservoir to get the idea of the production of resources and prevent the environmental pollution. We focused on how the pre-existing fractures propagate with the steady and non-steady injection pressures. In this study, especially we could adopt the multiple pre-existing fractures, which exist symmetrically around the borehole. To deal with the multiple fractures in the hydraulic fracturing simulation is much important to understand the realistic behavior of the fracture propagation. We simulated how the fractures could propagate with the steady and non-steady hydraulic pressure during the water injection. We could obtain two results from our numerical simulations: First, the curvature of the fracture trace depends on hydraulic pressure with the both cases of the steady and non-steady pressure, but no matter how the fluid pressure is, the orientation of fracture propagation finally converges to that of maximum principal stress. Second, the transition of the stress field follows the change of the injection pressure after some seconds, which means the delayed response of the fracture propagation in the non-steady hydraulic pressure condition. The simulation scheme of this study can deal with multiple fractures so that it could realize the quantitative estimation of fracture propagation in the complex stress region including various scales of fractures in the future.
\end{abstract}

\section{INTRODUCTION}

The reservoir developing with fracturing for the hydrothermal or shale oil and gas becomes popular in the world as the hydraulic fracturing method is improved. The fracture propagation from the injection well is strongly influenced by the regional stress around the borehole as well as the rock physical properties or reservoir formations. For the fracturing simulation, therefore, it is necessary to retrieve the whole stress distribution around the borehole one by one, which would change with the fracture propagation.

On the other hand, the hydraulic fracturing could cause some environmental issues; underground water pollution, induced seismicity, etc. It is expected to prevent such kind of environmental problem with hydraulic fracturing, so that we should estimate how fractures are created under various crustal state conditions to deal with unexpected behaviour of fracture propagation. For deriving the effective estimation of the pore pressure diffusion, crustal stress or fracture propagation, various approaches have been proposed. Adachi et al., (2007) adopted several basic equations such as the elasticity equation, the fluid flow equation, etc., and coupled them to govern a complicated hydraulic fracturing model. Finally it succeeded to estimate the area of fracture net around the injection well in the local stress field. Wangen (2011) represented fractures as fracture elements, which describe the fractures in the regular FEM-grid and use the strength of the bonds of the elements as a propagation criterion. They could make a physical model of the hydraulic fracturing including the fluid flow and simulate the fracture propagation in the practical scale. How the in-situ stress affects to the fracture propagation, however, was not discussed in these studies. Thus we focused the effects of the in-situ stress to the stress field around the fracture and the fracture propagation with both steady and non-steady hydraulic pressure conditions.

For the hydraulic fracturing simulation in crustal medium under the complicated stress field, we apply an extended finite element method (X-FEM) in this paper, which can retrieve the stress distribution affected by the fractures in the medium effectively and estimate the fracture propagation based on linear elastic fracture mechanics (LEFM). 
In this study we improved X-FEM for simulating the multiple fractures propagation at the same time to deal with the propagation of the pre-existing fractures around the borehole, which makes it possible to retrieve the stress distribution in the medium involving the multiple pre-existing fractures and simulate the fracture propagation symmetrically.

\section{METHODS}

\section{(1) X-FEM}

We apply X-FEM (Belytschko T. and T. Black, 1999) to consider the influence of fractures on the stress fields and simulate fracture propagation. This method saves us to calculate without remeshing associated with fracture propagation by adding secondary function into conventional FEM, which is called enrichment and the function is defined as the enrichment function. In the X-FEM, a displacement approximation for six nodes triangle elements is defined as follows:

$$
\boldsymbol{u}^{h}(\boldsymbol{x})=\sum_{I} \phi_{I}(\boldsymbol{x}) \boldsymbol{u}_{I}+\sum_{I \in C} \phi_{I}(\boldsymbol{x}) \sum_{k=1}^{4} \gamma_{k}(\boldsymbol{x}) \boldsymbol{a}_{I}^{k}+\sum_{I \in J} \phi_{I}(\boldsymbol{x}) H(\boldsymbol{x}) \boldsymbol{b}_{I}
$$

$\phi_{I}$ is a shape function used by classical FEMs. $C$ is the set of top nodes of finite elements, which includes crack tip, while $J$ is that which includes the intermediate part of the fracture. $\gamma_{k}$ is defined as

$$
\begin{gathered}
\gamma_{1}=\sqrt{r} \cos \frac{\theta}{2} \quad \gamma_{2}=\sqrt{r} \sin \frac{\theta}{2} \\
\gamma_{3}=\sqrt{r} \sin \frac{\theta}{2} \sin \theta \quad \gamma_{4}=\sqrt{r} \cos \frac{\theta}{2} \sin \theta
\end{gathered}
$$

where $(\boldsymbol{r}, \boldsymbol{\theta})$ are the local polar coordinates at the crack tip, and this expresses the stress concentration around the crack tip based on linear fracture mechanics. $H$ is also defined as

$$
H(x)=\left\{\begin{array}{cc}
1 & x \in \Omega_{+} \\
-1 & x \in \Omega_{-}
\end{array}\right\}
$$

This is a jump function so that we can take account of the discontinuity of stress distribution caused by the fracture cutting into two disjoint region, $\Omega_{+}$and $\Omega$.. To deal with multiple fractures, we should care the coordinates of the enrichment function $\gamma_{k}$ and the region $\Omega_{+}$and $\Omega$. in the jump function $H$ to unify the simulation of the fracture propagation.

\section{(2) PROPAGATION CRITERION}

We use the conventional energy-release rate approach of LEFM with J-integral (Rice, J.R., 1968) for the propagation criterion. The J-integral has a relationship with stress intensity factors (SIFs) as following

$$
J=\frac{K_{I}^{2}}{E^{*}}+\frac{K_{I I}^{2}}{E^{*}} \quad E^{*}=\frac{E}{1-v^{2}} \quad \text { (plane strain problem) }
$$

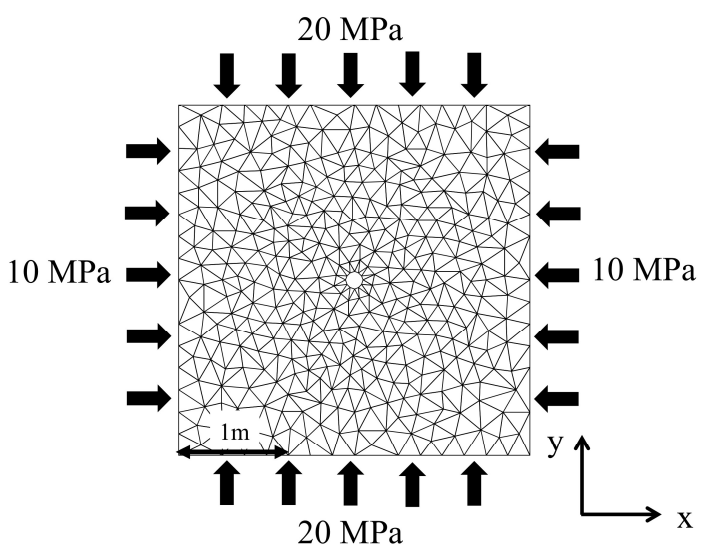

Figure 1 Simulation model. The maximum principal stress is fixed at $20 \mathrm{MPa}$ along y-axis while the minimum principal stress is also fixed at $10 \mathrm{MPa}$ along $\mathrm{x}$-axis.

where $E$ is Young's modulus and $v$ is Poisson ratio. There are two different modes of SIFs and it is difficult to obtain them separately. Therefore we use M-integral method (Yau, J.F., and S.S. Wang, 1984) to solve this problem. Two states are considered for M-integral. State 1 is the present state and state 2 is an auxiliary state. The M-integral for state 1 and 2 can be written

$$
M^{(1,2)}=\int_{\Gamma}\left[W^{(1,2)} \delta_{1 j}-\sigma_{i j}^{(1)} \frac{\partial u_{i}^{(2)}}{\partial x_{j}}-\sigma_{i j}^{(2)} \frac{\partial u_{i}^{(1)}}{\partial x_{j}}\right]
$$

where $W$ is strain energy, $\delta_{1 \mathrm{j}}$ is defined as $\delta_{11}=$ $1, \delta_{12}=0$ and $\sigma_{\mathrm{ij}}$ is stress tensor. $\Gamma$ is arbitrary integralation pass around a crack tip.

The equation between M-integral and SIFs is

$$
M^{(1,2)}=\frac{2}{E^{*}}\left(K_{I}^{(1)} K_{I}^{(2)}+K_{I I}^{(1)} K_{I I}^{(2)}\right)
$$

where we can obtain $K_{I}$ and $K_{I I}$ by choosing appropriate values of auxiliary state i.e. $K_{I}^{(2)}=1$, and $K_{I I}^{(2)}=0$.

The orientation $\theta$ of fracture propagation is estimated based on maximum circumferential stress criterion (Erdogan, F., and G.C. Sih, 1963). The velocity of fracture propagation and the interval of the stress recovery from the stress drop caused by the propagation are set uniformly for the kinetic simulation.

\section{SIMULATION MODEL}

Figure 1 shows the two-dimensional simulation model. We use the triangle quadratic element. The number of finite element is 729 and the model scale is $4 \mathrm{~m} \times 4 \mathrm{~m}$. The borehole diameter is $0.2 \mathrm{~m}$. The Young's modulus of the background rock mass is $15 \mathrm{GPa}$ and the Poisson's ratio is 0.3 . The maximum 

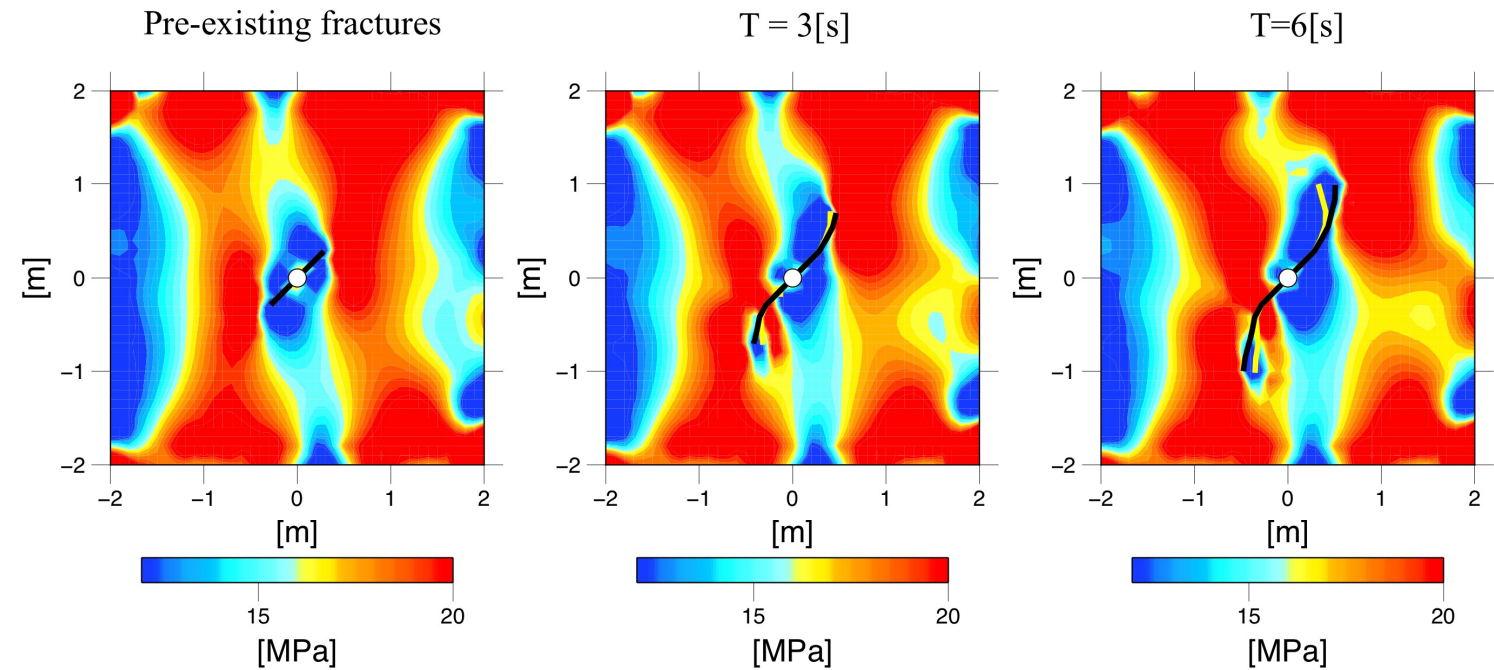

Figure 2 Left: the arrangement of the symmetrical pre-existing fractures. Black line shows the fractures and color contour shows the Mises stress. Middle and Right: the fracture propagation at $\mathrm{T}=3[\mathrm{~s}]$ and $\mathrm{T}=6[\mathrm{~s}]$. Black line shows with hydraulic pressure $20 \mathrm{MPa}$ while yellow line shows with $12 \mathrm{MPa}$.

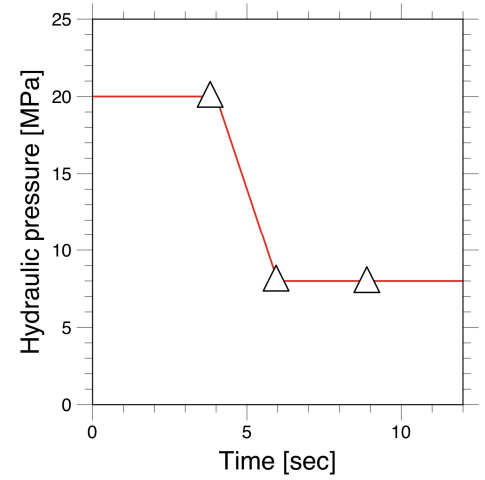

Figure 3 Hydraulic pressure rate. Triangles show the time and hydraulic pressure corresponding to Figure 4.
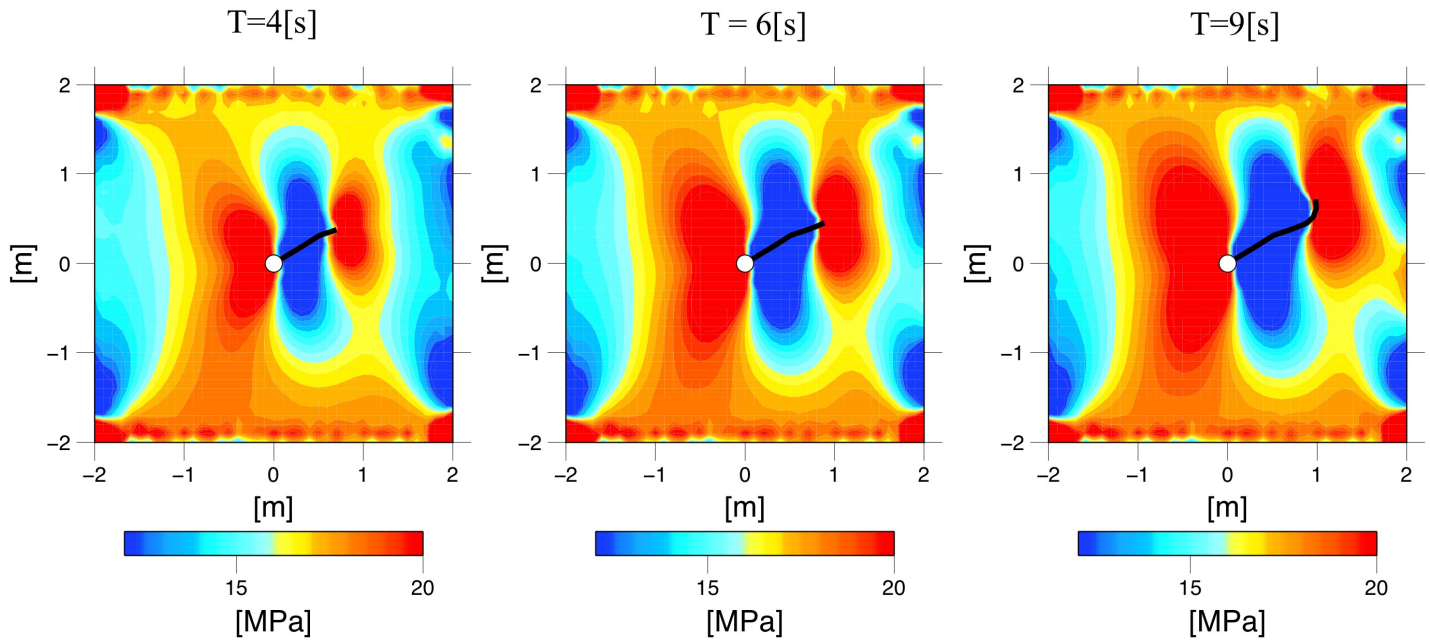

Figure 4 Fracture propagation with non-steady hydraulic pressure at each time in Figure 3.

principal stress is fixed at $20 \mathrm{MPa}$ along y-axis and the minimum principal stress is at $10 \mathrm{MPa}$ along $\mathrm{x}$-axis as in-situ stress. These principal stresses do not change during fracture propagation. We put pre-existing fractures symmetrically and simulate the propagation of these fractures.

\section{RESULTS AND DISCUSSIONS}

(1) FRACTURE PROPAGATION WITH STEADY HYDRAULIC PRESSURES

First result is for the fracture propagation under two different steady hydraulic pressures. Figures 2 
shows the results of the simulation in which the hydraulic pressure is set as constant $20 \mathrm{MPa}$ and $12 \mathrm{MPa}$ respectively. The color contour means Mises stress.

These results show that the convergence speed to the principal stress orientation could be inversely correlated to as the hydraulic pressure. The fracture propagation turns to the orientation of the maximum principal stress relatively more quickly in $12 \mathrm{MPa}$ (Figure 2 by yellow line) than the case in $20 \mathrm{MPa}$ (Figure 2 by black line). It is also found that these two pre-existing fractures propagate to the maximum principal stress orientation symmetrically, which confirms the relationship between the fracture propagation and the hydraulic pressure.

In addition, the orientation of the fracture propagation finally converges finally to the direction of the maximum principal stress at any hydraulic pressure. This result suggests that the direction of the in-situ maximum principal stress, rather than the initial direction of the fracture, is dominant on fracture propagations. This characteristic of the fracture propagation corresponds to the observational fact that fractures in hydraulic fracturing using horizontal well tend to propagate to the vertical direction, which is the maximum principal stress orientation.

\section{(2) FRACTURE PROPAGATION WITH NON-STEADY HYDRAULIC PRESSURES}

Second result is the simulation of the fracture propagation under the non-steady hydraulic pressure during the injection. We set $20 \mathrm{MPa}$ as the hydraulic pressure during the first 4 seconds, then down to $8 \mathrm{MPa}$ gradually as Figure 3 . There are three figures of fracture propagation at each time indicated in Figure 4.

This result indicates that the convergence speed could be inversely related to the hydraulic pressure. It is shown that the tendency of the fracture propagation with non-steady hydraulic pressure is same as the fracture propagation we observed in the steady hydraulic pressure condition.

We also found a time delay between the change of the hydraulic pressure and the transition of the stress distribution, which leads to the delayed turning of the fracture propagation orientation. Although the hydraulic pressure starts to gradually decrease at $4 \mathrm{~s}$ down to $8 \mathrm{MPa}$, the fracture still propagates straight (around $6 \mathrm{~s}$; see Figure 4 middle) and then turn to the direction of the maximum principal stress several seconds after the hydraulic pressure has completely been decreased. From this result it could be suggested that the local stress distribution changes due to the hydraulic pressure change with a time delay, which might cause the delay of the turning to the direction of the maximum principal stress.

We expected that this time delay becomes significant with the large spatial scale. Therefore for the real operation we should estimate this time delay quantitatively and apply to the fracture propagation prediction.

\section{CONCLUSIONS}

We would like to conclude as follows: 1) The fractures turn with different trace correspond to the hydraulic pressure, but no matter how the hydraulic pressure is, the orientation of fracture propagation finally converges to that of maximum principal stress. The two symmetric pre-existing fractures show much same behavior. 2) There is a time delay from the change of the hydraulic pressure to the transition of the stress distribution, which causes the delayed response of the fracture propagation in the non-steady hydraulic pressure condition.

This method could be applied to the more multiple fractures propagation including various sizes of fractures. For the future work, it also should be considered to interfere with each fracture.

\section{REFERENCES}

1)Adachi, J., E. Siebrits, A. Peirce, and J. Desroches, 2007. Computer simulation of hydraulic fractures, International Journal of Rock Mechanics \& Mining Sciences, 44, 739-757.

2)Wangen, M., 2011. Finite element modeling of hydraulic fracturing on a reservoir scale in 2D, Journal of Petroleum Science and Engineering, 77, 274-285.

3)Belytschko, T. and T. Black, 1999. Elastic crack growth in finite elements with minimal remeshing, Int. J. Numer. Meth. Eng., 45, 601-620.

4)Rice, J. R., 1968. A Path Independent Integral and the Approximate Analysis of Strain Concentration by Notches and Cracks, Journal of Applied Mechanics, 35, 379-386.

5)Yau, J.F., and S.S. Wang, 1984. An analysis of interface cracks between dissimilar isotropic materials using conservation integrals in elasticity, Engineering Fracture Mechanics, 20 (3), 423-432.

6)Erdogan, F. and G.C. Sih, 1963. On the crack extension in plates under plane loading and transverse shear Transactions of the ASME, Journal of Basic Engineering, 85D, 519-527. 